\title{
Vorticity in the solar photosphere
}

\author{
S. Shelyag, P. Keys, M. Mathioudakis, and F. P. Keenan
}

\author{
Astrophysics Research Centre, School of Mathematics and Physics, Queen's University Belfast, Belfast, BT7 1NN, \\ Northern Ireland, UK \\ e-mail: s.shelyag@qub.ac.uk
}

Received 26 August 2010 / Accepted 27 October 2010

\begin{abstract}
Aims. We use magnetic and non-magnetic 3D numerical simulations of solar granulation and G-band radiative diagnostics from the resulting models to analyse the generation of small-scale vortex motions in the solar photosphere.

Methods. Radiative MHD simulations of magnetoconvection are used to produce photospheric models. Our starting point is a nonmagnetic model of solar convection, where we introduce a uniform magnetic field and follow the evolution of the field in the simulated photosphere. We find two different types of photospheric vortices, and provide a link between the vorticity generation and the presence of the intergranular magnetic field. A detailed analysis of the vorticity equation, combined with the G-band radiative diagnostics, allows us to identify the sources and observational signatures of photospheric vorticity in the simulated photosphere.

Results. Two different types of photospheric vorticity, magnetic and non-magnetic, are generated in the domain. Non-magnetic vortices are generated by the baroclinic motions of the plasma in the photosphere, while magnetic vortices are produced by the magnetic tension in the intergranular magnetic flux concentrations. The two types of vortices have different shapes. We find that the vorticity is generated more efficiently in the magnetised model. Simulated G-band images show a direct connection between magnetic vortices and rotary motions of photospheric bright points, and suggest that there may be a connection between the magnetic bright point rotation and small-scale swirl motions observed higher in the atmosphere.
\end{abstract}

Key words. Sun: photosphere - Sun: surface magnetism - plasmas - magnetohydrodynamics

\section{Introduction}

One of the consequences of turbulent plasma movement in the solar photosphere is the appearance of horizontal vortex motions. These motions can be of significance for the generation of magnetohydrodynamic (MHD) waves which propagate to the upper layers of the solar atmosphere (Parker 1988; Fedun et al. 2009; Jess et al. 2009). As a result of recent advancements in state-of-the-art instrumentation and observational techniques, it has now become possible to observe small-scale vortices in the lower solar atmosphere.

Bonet et al. (2008) showed the presence of vortex motions in photospheric G-band bright points with lifetimes comparable to those of the granules. Wedemeyer-Böhm \& Rouppe van der Voort (2009); Wedemeyer-Böhm (2010) performed simultaneous G-band and Ca II $8542 \AA$ imaging to demonstrate the presence of small-scale swirl motions in the chromosphere. Carlsson et al. (2010) also demonstrate a presence of the chromospheric swirls in their simulations, which include the chromospheric layer.

A thorough investigation of the non-magnetic photospheric convection by Stein \& Nordlund (1998) has shown the generation of vorticity by baroclinic fluid motions in the upper convection zone. Such motions, characterised by large and nonparallel gradients of density and pressure, occur near the edges of granules. Some studies have also been performed on the vorticity generated by magnetic flux tubes rising to the solar surface from the deep sub-photosphere (e.g. Emonet \& MorenoInsertis 1998; Emonet et al. 2001). The link between vortex generation and magnetic field in the upper photosphere was also noted by Vögler et al. (2005). Generation of Alfvénic vortices by the interaction of compressible plasma with field-aligned obstacles was studied by Gruszecki et al. (2010). Recently, Kitiashvili et al. (2010) used numerical simulations to demonstrate the significance of vortex motions and vortex dragging for the creation of pore-like magnetised structures in the photosphere.

Here we present a more detailed study of the photospheric vorticity. We focus on its origins and connection to the photospheric magnetic field and granulation dynamics. We show a direct correspondence between the photospheric vortices that correspond to G-band bright point motions, with the strong intergranular magnetic field. We also demonstrate a presence of vortex motions in the upper atmosphere, which may be connected to the chromospheric swirls.

In Sect. 2 we provide a brief description of the numerical model, simulation setup and the results obtained. The output of the simulations is analysed in terms of the vorticity equation in Sect. 3. Section 4 describes the radiative diagnostics and observational consequences of vortex motions in the photosphere, while in Sect. 5 we summarise our conclusions.

\section{Simulations}

We use the MuRAM code (Vögler et al. 2005) to perform the simulations, which has been successfully used for a wide range of solar applications (Schüssler et al. 2003; Shelyag et al. 2004, 2007; Cheung et al. 2008; Pietarila Graham et al. 2009; Rempel et al. 2009; Yelles Chaouche et al. 2009; Danilovic et al. 2010). The code solves large-eddy radiative three-dimensional MHD equations on a Cartesian grid, and employs a fourth-order central difference scheme to calculate spatial derivatives. A fourthorder Runge-Kutta scheme is used to advance the numerical 


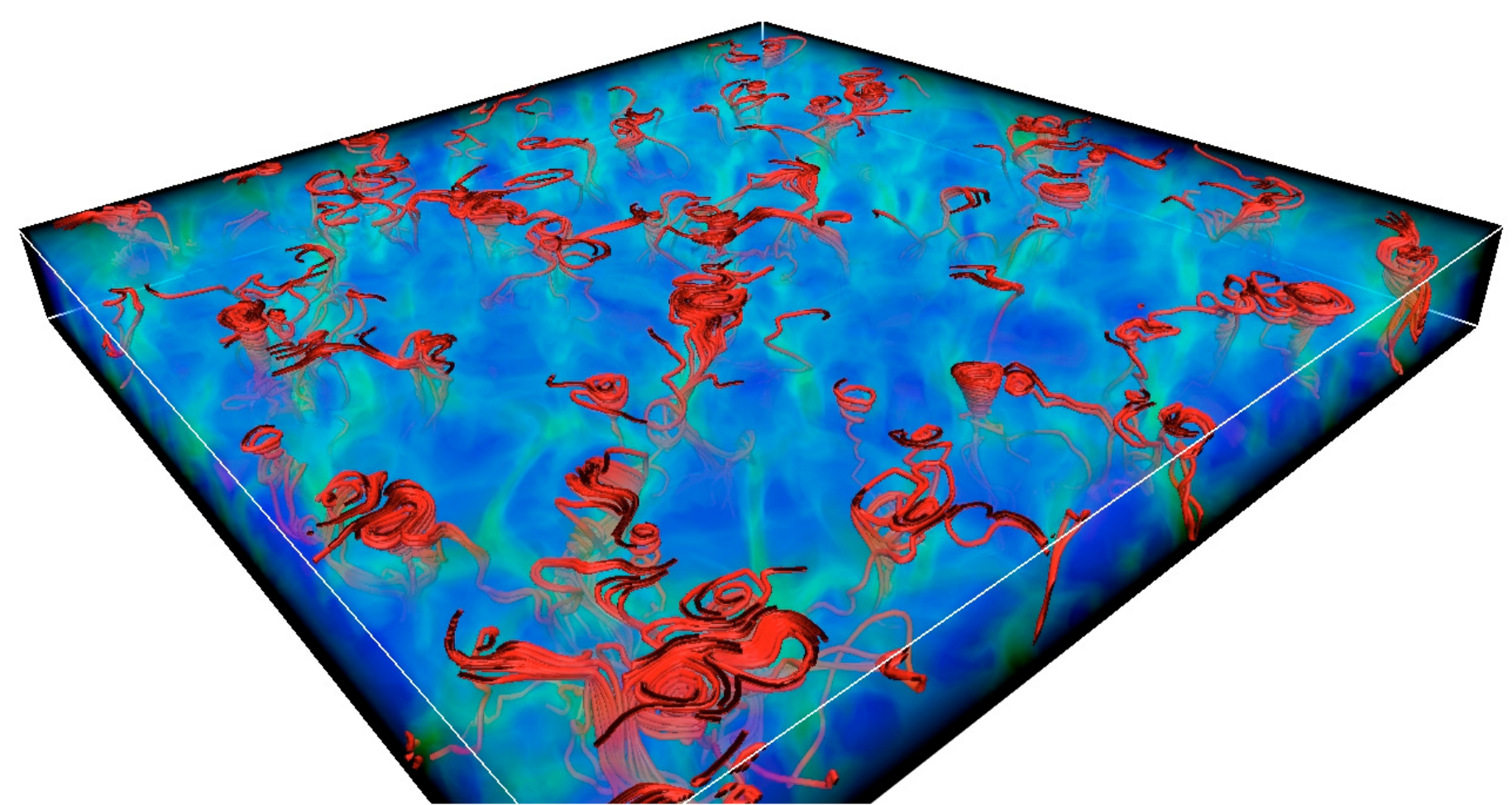

Fig. 1. Geometry of the domain. Stream lines (red) are plotted over the three-dimensional structure of the vertical component of the magnetic field (blue-green).

solution in time. Hyperdiffusivity sources are used to stabilise the solution against numerical instabilities and to account for physical processes which are not resolved by the numerical grid. Non-grey radiative energy transport is included in the code using short-characteristics and opacity binning techniques. A non-ideal equation-of-state, taking into account the first-stage ionisation of the 11 most abundant elements in the Sun, is also included.

The numerical domain has a physical size of $12 \times 12 \mathrm{Mm}^{2}$ in the horizontal direction, $1.4 \mathrm{Mm}$ in the vertical direction $(y)$, and is resolved by $480 \times 480$ and 100 grid cells, respectively. The lower boundary is transparent, allowing the plasma to travel in and out of the domain. The upper boundary of the domain is closed, however, it allows the horizontal motions of the plasma and the magnetic field lines. The side boundaries of the domain are set to be periodic. The domain is positioned in such a way that the visible solar surface ${ }^{1}$ is located approximately $600 \mathrm{~km}$ below the upper boundary.

Our starting point for the simulations is a well-developed non-magnetic $(\boldsymbol{B}=0)$ snapshot of photospheric convection taken at $t \sim 2000 \mathrm{~s}$ (about 8 convective turnover timescales) from the initial plane-parallel model. A uniform vertical magnetic field of $B_{y}=200 \mathrm{G}$ has been introduced at this stage, and a sequence of 147 snapshots recorded, containing physical parameters of the model, such as velocity and magnetic field vectors, temperature, density, pressure and internal energy. The sequence covers approximately $40 \mathrm{~min}$ of physical time, corresponding to 5-10 granular lifetimes. During the simulation, the magnetic field is advected into the intergranular lanes by the convective

\footnotetext{
${ }^{1}$ In this paper we refer to the visible solar surface as a horizontal geometrical layer which is physically close to the optical layer of radiation formation. We assume that the geometrical properties of the analysed features do not significantly change within this layer.
}

plasma motions, and the maximum field strength rises from its initial value of $200 \mathrm{G}$ to a few kilogauss in the intergranular lanes. The sequence we obtained is long enough for the relaxation of the model, since the time needed for the magnetic field redistribution is about $0.1-0.2 \mathrm{~h}$ (Vögler et al. 2005; Vögler \& Schüssler 2007).

A three-dimensional rendering of the velocity and magnetic field is shown in Fig. 1. The image is generated with the VAPOR $3 \mathrm{D}$ visualisation package (Clyne et al. 2007). Stream lines (red curves) reveal a large amount of vortex motions in the upper photosphere. These vortices appear primarily in the intergranular lanes and coincide with regions of strong magnetic fields and downflows.

The modulus of the horizontal velocity components is shown in Fig. 2, where the top panels correspond to a level close to the visible solar surface level, and the bottom ones to a height in the domain close to the temperature minimum. Images on the left correspond to the initial non-magnetic model, and those on the right to the fully developed magnetic snapshot. It is evident from the figure that small-scale vortex structures have formed in the magnetised model in the upper photosphere (bottom-right panel). These structures are not seen in the non-magnetic model nor at the visible solar surface. The contours in the bottom-right panel of Fig. 2, which bound the granular regions where the vertical component of magnetic field $B_{y}<30 \mathrm{G}$, clearly demonstrate that the vortices in the upper photosphere are co-spatial with the magnetic field concentrations in the intergranular network.

\section{The vorticity equation}

Vorticity is defined as the curl of velocity, $\omega=\nabla \times \boldsymbol{v}$. Similar to Stein \& Nordlund (1998); Moreno-Insertis \& Emonet (1996); Emonet \& Moreno-Insertis (1998); Emonet et al. (2001), we 

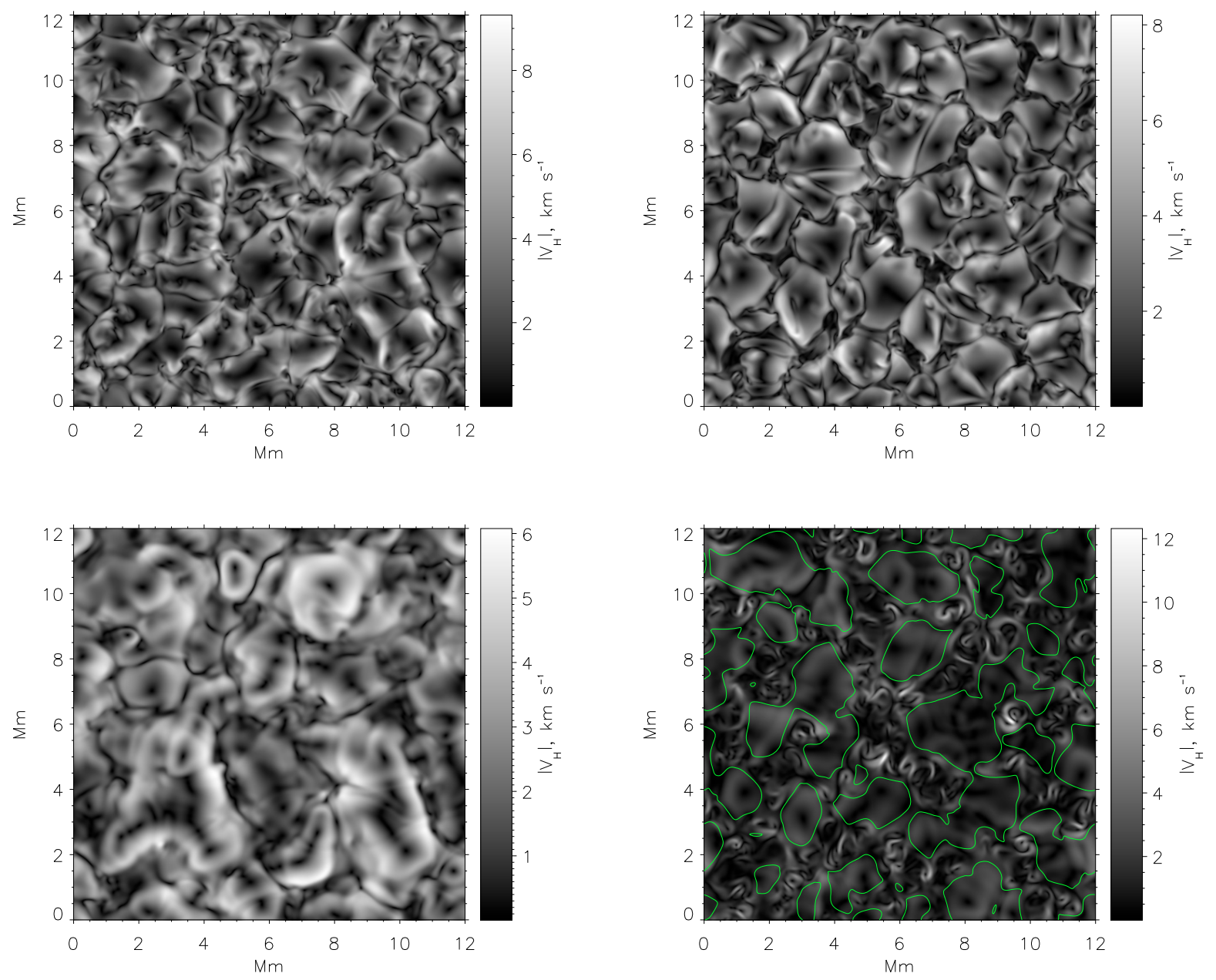

Fig. 2. Horizontal cuts of the modulus of the horizontal components of velocity in the domain. The cuts are taken approximately at the visible solar surface (top panels) and in the upper photosphere (bottom panels). The left panels correspond to the initial non-magnetic snapshot, while the right panels correspond to the well-developed magnetic model. Vortex motions are clearly visible in the bottom-right panel. The contours on the bottom-right panel bound the regions where the vertical component of magnetic field $B_{y}<30 \mathrm{G}$.

write the vorticity equation as the curl of the momentum equation of the MHD system:

$\rho \frac{\mathrm{D}}{\mathrm{D} t} \frac{\omega}{\rho}=(\omega \cdot \nabla) \boldsymbol{v}-\nabla \frac{1}{\rho} \times \nabla p_{\mathrm{g}}+\nabla \times\left[\frac{1}{\rho} \boldsymbol{J} \times \boldsymbol{B}\right]$.

By substituting the current vector $\boldsymbol{J}$ with $\nabla \times \boldsymbol{B}$ and combining the magnetic pressure term with the gas pressure, we obtain the following equation:

$\rho \frac{\mathrm{D}}{\mathrm{D} t} \frac{\omega}{\rho}=(\omega \cdot \nabla) \boldsymbol{v}-\nabla \frac{1}{\rho} \times \nabla\left(p_{\mathrm{g}}+p_{\mathrm{m}}\right)+\nabla \times \frac{1}{\rho}(\boldsymbol{B} \cdot \nabla) \boldsymbol{B}$,

where $\mathrm{D} / \mathrm{D} t$ represents the full (material) derivative, $\omega$ is the vorticity vector, $\rho$ and $p_{\mathrm{g}}$ are the plasma density and pressure, $\boldsymbol{v}$ is the velocity vector, $\boldsymbol{B}$ is the magnetic field vector, and $p_{\mathrm{m}}=\boldsymbol{B}^{2} / 2$ is the magnetic pressure. Note that the magnetic field is normalised by a factor $\sqrt{4 \pi}$ for convenience. Viscous dissipation of vorticity has been neglected in this equation. The material derivative in the left side of Eq. (1) is expressed in terms of Euler derivatives as:

$\rho \frac{\mathrm{D}}{\mathrm{D} t} \frac{\omega}{\rho}=\frac{\partial \omega}{\partial t}+\omega(\nabla \cdot \boldsymbol{v})+(\boldsymbol{v} \cdot \nabla) \omega$

Equation (2) can be rewritten by separating the magnetic and non-magnetic terms and decomposing the last magnetic term into two:

$$
\begin{aligned}
\rho \frac{\mathrm{D}}{\mathrm{D} t} \frac{\omega}{\rho}= & \overbrace{(\omega \cdot \nabla) \boldsymbol{v}}^{T_{1}}-\overbrace{\nabla \frac{1}{\rho} \times \nabla p_{\mathrm{g}}}^{T_{2}} \\
& \overbrace{-\nabla \frac{1}{\rho} \times\left[\nabla p_{\mathrm{m}}-(\boldsymbol{B} \cdot \nabla) \boldsymbol{B}\right]}^{\left.T_{3}\right]}+\overbrace{\frac{1}{\rho} \nabla \times[(\boldsymbol{B} \cdot \nabla) \boldsymbol{B}]}^{T_{4}} .
\end{aligned}
$$

The right-hand side of Eq. (4) shows the different physical mechanisms associated with the generation of vorticity. $T_{1}$ is the vortex tilting term, $T_{2}$ is responsible for the hydrodynamic baroclinic vorticity generation, $T_{3}$ represents the magnetic baroclinic vorticity, and $T_{4}$ corresponds to vorticity generated by the magnetic tension. The quantity in square brackets within $T_{3}$ represents the deviation of the magnetic field configuration from a potential field.

Horizontal cuts of the vertical component of vorticity are shown in Fig. 3. The upper panels show the vorticity maps at a level close to the visible solar surface, while the lower panels correspond to the upper photosphere. Non-magnetic and magnetic snapshots are shown in the left and right panels, respectively.

In the non-magnetic case, the vorticity is generated by the hydrodynamic baroclinic term and is mostly randomly directed 

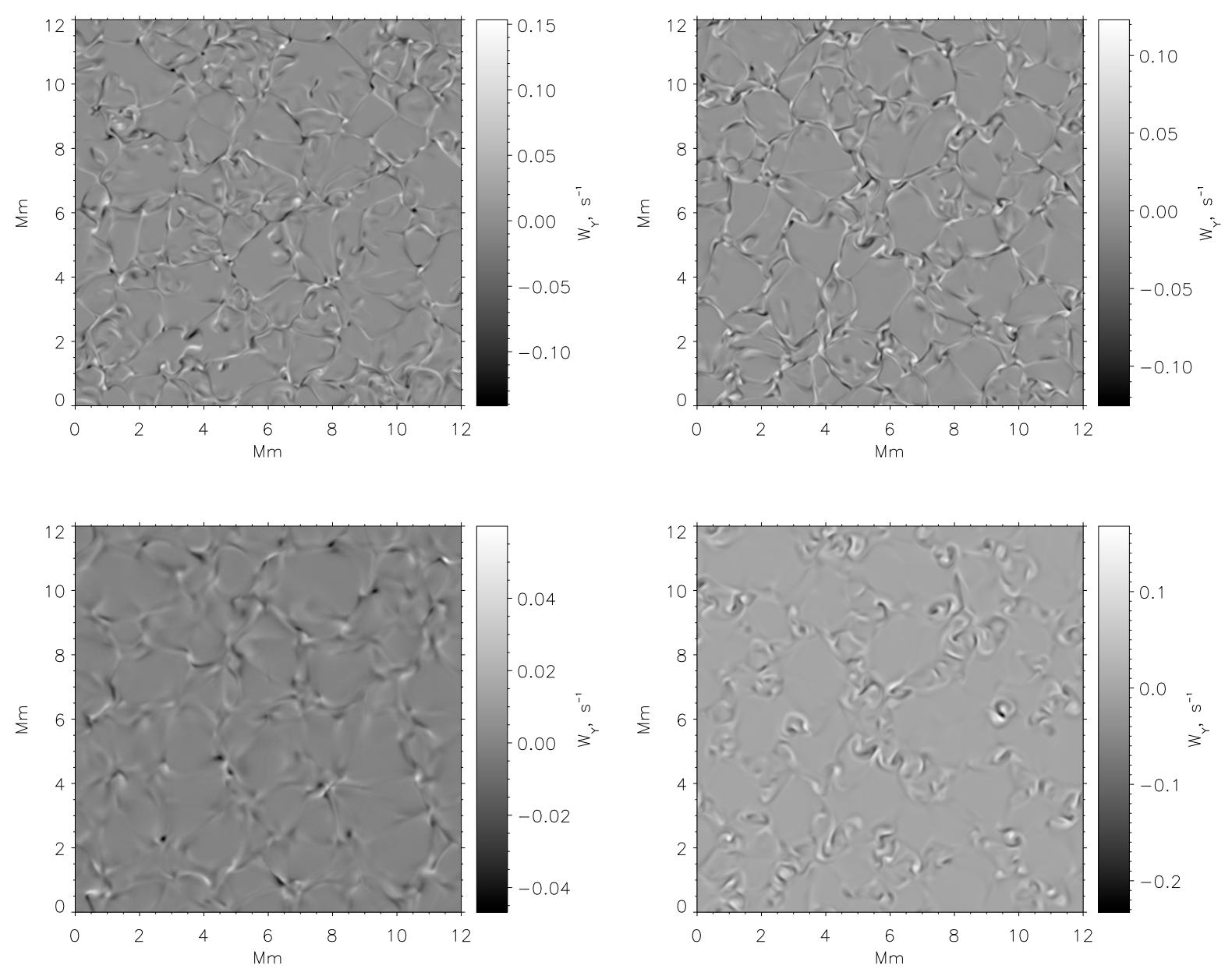

Fig. 3. Vertical component of vorticity $\omega_{y}$ at the visible solar surface (upper panels) and in the upper photosphere (lower panels). Non-magnetic (left) and magnetic (right) snapshots are shown.

without any internal structure of the vortices in the intergranular lanes. However, we notice a change in the structure of the vortices after we incorporated the magnetic field into the numerical box. Once the magnetic field has been advected into the intergranular network, both positive and negative polarities of the vorticity coexist within a vortex, with both clockwise and counterclockwise motions appearing. An increase in the amplitude of the vertical component of vorticity by a factor of about 5 is also observed.

In order to determine the origin of the vorticity, the terms in Eq. (4) need to be analysed separately for the whole simulation sequence. The vertical component of vorticity, which corresponds to the horizontal vortex motions, is of primary interest. Thus, a mean value of the modulus of the vertical vorticity component is a good measure of the amount of vertical vorticity generated in the model.

Figure 4 shows the evolution of the mean of the modulus of the vertical vorticity component at different heights in the domain, together with the time dependences of the $y$-components of $T_{1}$ through $T_{4}$ terms of Eq. (4). The left panels correspond to the upper photosphere $(500 \mathrm{~km}$ above the visible solar surface), the middle panels the approximate level of the visible solar surface, and the right panels correspond to the convection zone ( $650 \mathrm{~km}$ below the visible solar surface).

An inspection of the left panels of Fig. 4 reveals that the vorticity in the upper layers of the model is produced by the magnetic field, rising from its non-magnetic value of $0.0025 \mathrm{~s}^{-1}$ to $0.01 \mathrm{~s}^{-1}$ within the first three minutes of the simulation. During this phase, the magnetic field gets almost completely transported into the intergranular lanes by convective motions of plasma (Vögler et al. 2005). The amount of vorticity produced in the photosphere (middle-top plot) remains roughly the same, experiencing some initial decrease, which may be connected to the suppression of plasma motions by the initially uniform magnetic field, before it increases again at $t=2-4 \mathrm{~min}$. The behaviour of the vorticity at the bottom of the domain is opposite to what is observed at the top: in the first 5 min of the simulation, the amount of vorticity has decreased by almost a factor of 2 .

The evolution of vorticity shown in Fig. 4 can be explained by analysing the relative importance of the vertical components of the $T_{1}$ through $T_{4}$ terms in Eq. (4). In the upper photosphere, the term which corresponds to the vorticity generation by the magnetic tension $\left(T_{4}\right.$, solid black line) experiences a sharp rise, and after the initial phase of the simulation ( $4 \mathrm{~min})$ takes the largest value among the other terms. The same behaviour is observed for the magnetic baroclinic term $T_{3}$ (red dashed line), with an amplitude which is a factor of 3 smaller than that of $T_{4}$. The hydrodynamic baroclinic term $T_{2}$ (blue dash-dotted line) is a factor of 2 smaller than $T_{3}$. Thus, the baroclinic motions of the fluid (in the hydrodynamical sense) do not make a significant impact on the vortex generation in the upper photosphere.

A different picture is observed at the photospheric level. Here, the amount of vorticity produced by the hydrodynamic baroclinic motions of the fluid is similar to the amount of vorticity, generated by the magnetic tension. In the initial stage of the simulation, $T_{4}$ and $T_{2}$ behave in an opposite way. This is caused 

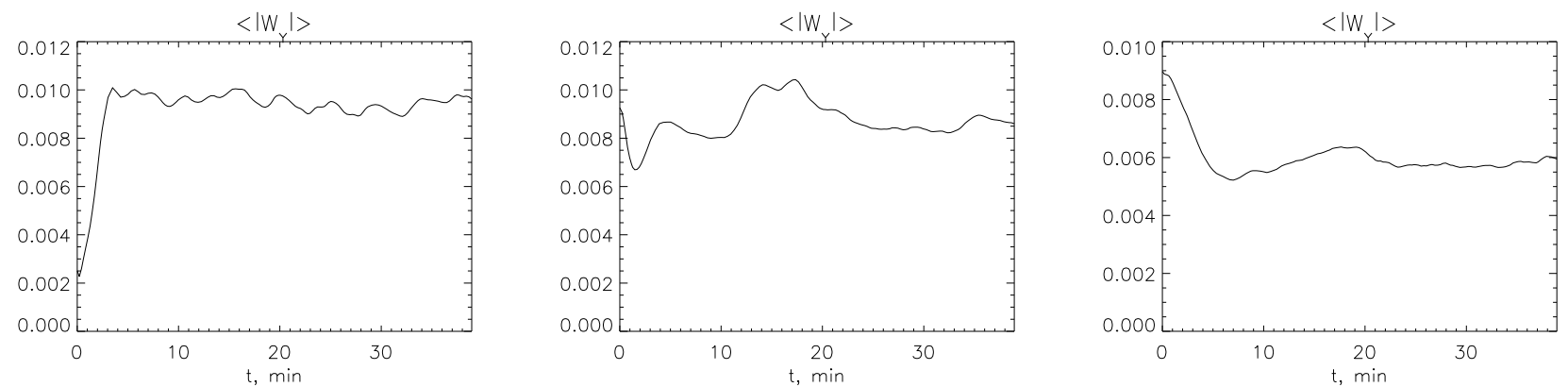

$<\left|T_{Y}\right|>$
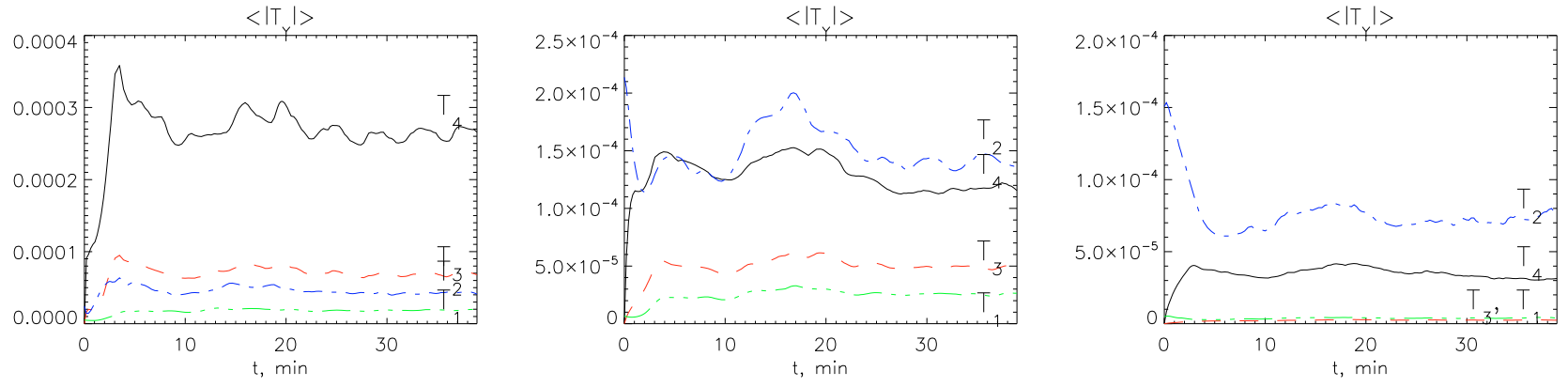

Fig. 4. Dependence of $\left\langle\left|\omega_{y}\right|\right\rangle$ (top plots) and of different terms of the vorticity equation (bottom plots) on time at different heights in the domain. The first column corresponds to the upper photosphere (500 km above the approximate visible solar surface level), the second column is the visible surface level, and the third column corresponds to the convection zone (650 km below the visible surface level).

by the processes of magnetic field redistribution. Initially, the magnetic field is uniform, and suppresses the plasma motions both in the granules and in the intergranular lanes, thus decreasing the vorticity generation by hydrodynamic baroclinic term $T_{2}$, while $T_{4}$ experiences a sharp increase due to the formation of the magnetic flux concentrations. After the initial stage, when the convection pushes the magnetic field out of the granules, both the magnetic-type vortices and hydrodynamic vortices can be produced.

In the convection zone, (right panels in Fig. 4), where the plasma $\beta$ is high, the hydrodynamic baroclinic term $T_{2}$ is the primary source of vorticity. Although $T_{2}$ decreases significantly from its non-magnetic value within the first few minutes of the simulation, it remains a factor of 2 larger than the magnetic tension term $T_{4}$.

We note that $T_{4}$ plays a significant role for the vorticity generation both in the convection zone, where the plasma $\beta$ is large, and in the upper photosphere, where plasma $\beta$ is small in the magnetic field concentrations. This term has no "hydrodynamic" equivalent and represents a physical mechanism for vorticity generation which is separate in nature from the conventional baroclinic vorticity generation processes. The magnetic baroclinic term $T_{3}$ does not show any significant influence on the generation of vorticity. The significance of the hydrodynamic baroclinic vorticity generation term increases with depth.

$3 \mathrm{mHz}$ acoustic oscillations are also present in the domain. Both the vertical component of vorticity $\omega_{y}$ and $T_{4}$ show signs of these oscillations (see upper left plot of Fig. 4) after the simulation has passed its initial stage. This finding, together with the connection of the photospheric vortices to the magnetic field, confirms the idea that the oscillations leak through magnetic concentrations to the upper photosphere. An observational search for oscillatory signals in vorticity may be possible.

\section{Radiative diagnostics}

Photospheric bright points correspond to regions of strong intergranular magnetic fields (Schüssler et al. 2003; Carlsson et al. 2004; Shelyag et al. 2004) and may be subject to vortex motions.
Recent high spatial resolution observations indicate that this may indeed be the case (Bonet et al. 2008). The analysis we present includes the radiative diagnostics in the G-band. Using the methods described by Frutiger (2000), Berdyugina et al. (2003) and Shelyag et al. (2004), we have computed G-band images for all 147 sequential simulation snapshots. The direct effect of the magnetic field on the absorption line profiles has not been included in the calculations. The sequence of images allowed us to not only to find and track the vortex motions of the photospheric G-band bright points, but also to study their appearance during the initial stages of the simulation while the magnetic field was being advected to the intergranular lanes. An inspection of the images made possible to identify vortex motions associated with magnetic bright points (MBPs) in the simulated photosphere. An example is shown in Fig. 5 and confirms the connection between the photospheric vortex motions, rotation of MBPs and vorticity in the upper layers of the simulated photosphere. Three consecutive simulated G-band images of an MBP (top panels), together with vorticity maps at a height corresponding to the visible solar surface (middle) and in the upper photosphere (bottom) are shown in the figure. All images are taken at the same horizontal position. The evolutionary track of the MBP is very similar to the observations of Bonet et al. (2008). The bottom images clearly resemble the evolution of chromospheric swirl (Wedemeyer-Böhm \& Rouppe van der Voort 2009), despite the data being obtained somewhat lower than the level of Ca II core formation. This fact suggests that the chromospheric swirls may be connected to the vortices, generated in the solar magnetic photosphere, however, a further investigation is needed to provide a rigorous proof for that.

\section{Conclusions}

In this paper, we have examined the processes leading to vorticity generation in the simulated magnetised photosphere. We have shown that large amount of vorticity in the photosphere is formed as a result of the photospheric plasma interaction with the magnetic field in the intergranular lanes. The amount of 

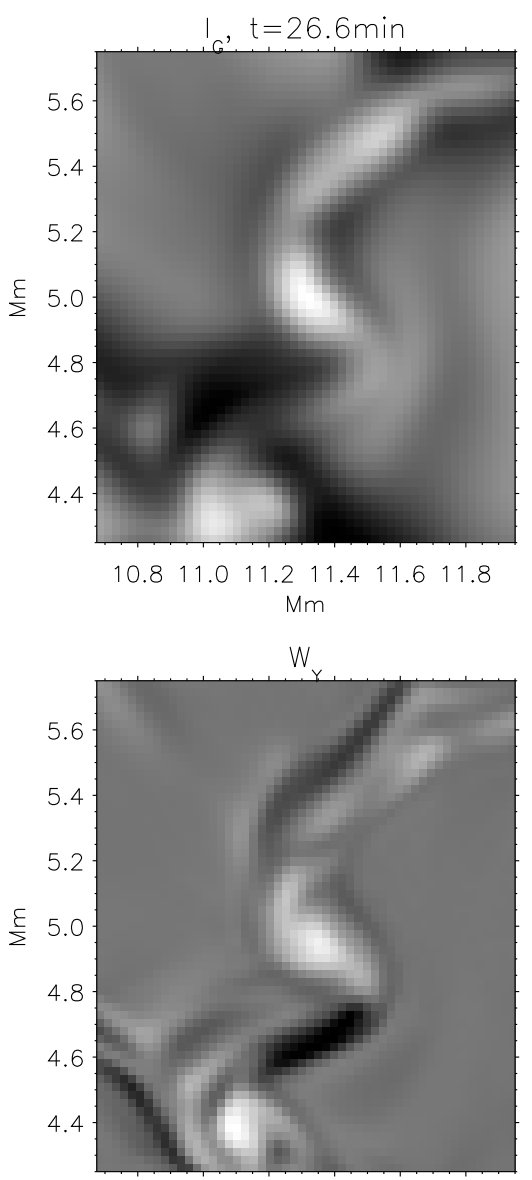

10.811 .011 .211 .411 .611 .8 $\mathrm{Mm}$

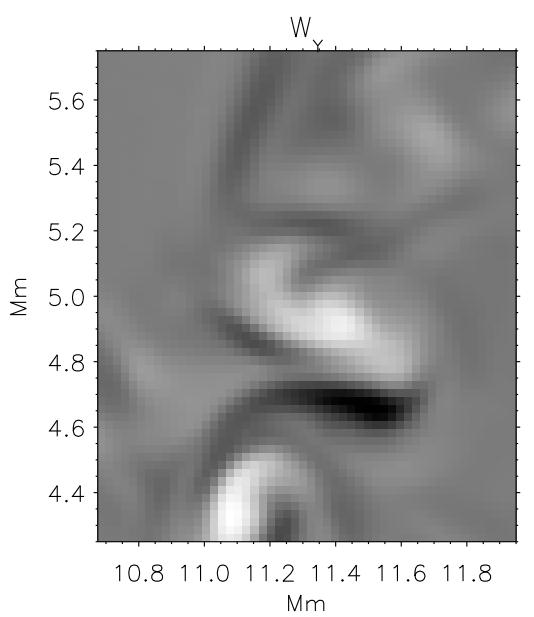

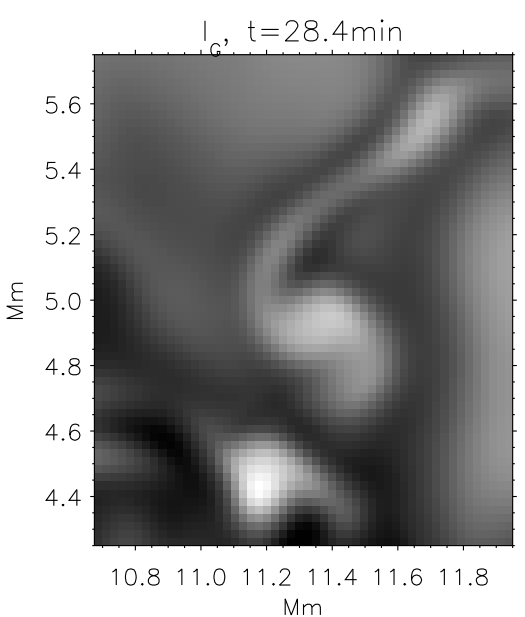

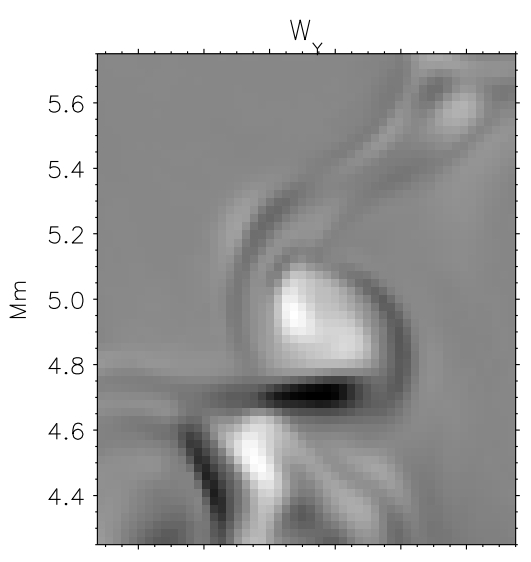

10.811 .011 .211 .411 .611 .8 $\mathrm{Mm}$

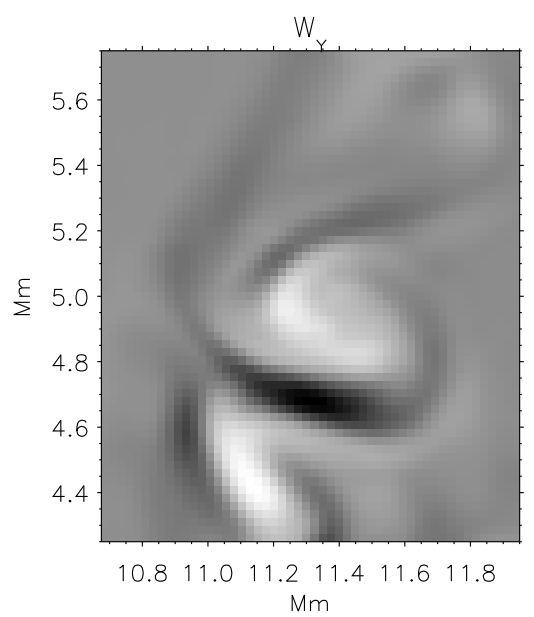

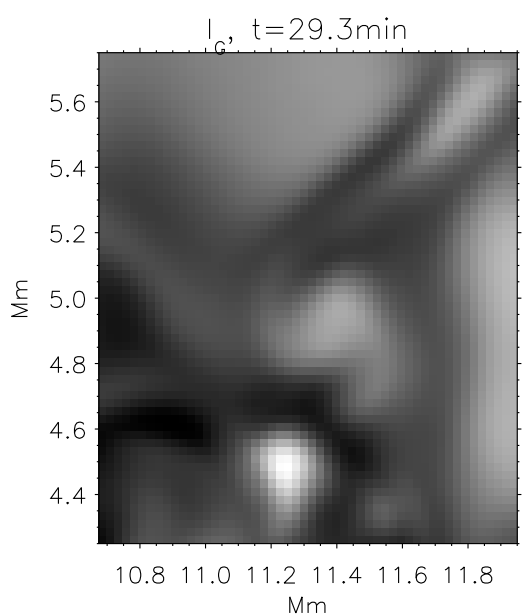

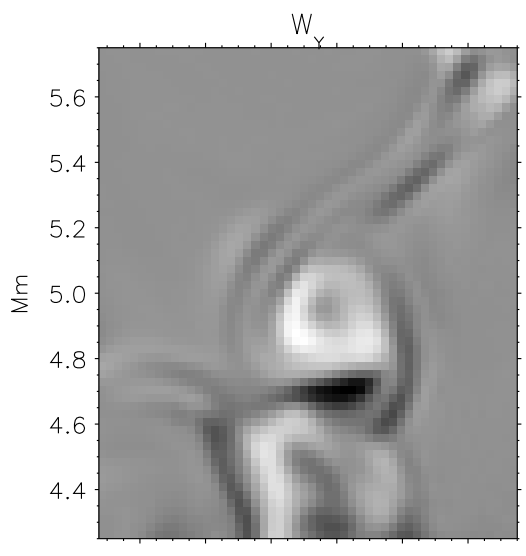

10.811 .011 .211 .411 .611 .8 $\mathrm{Mm}$

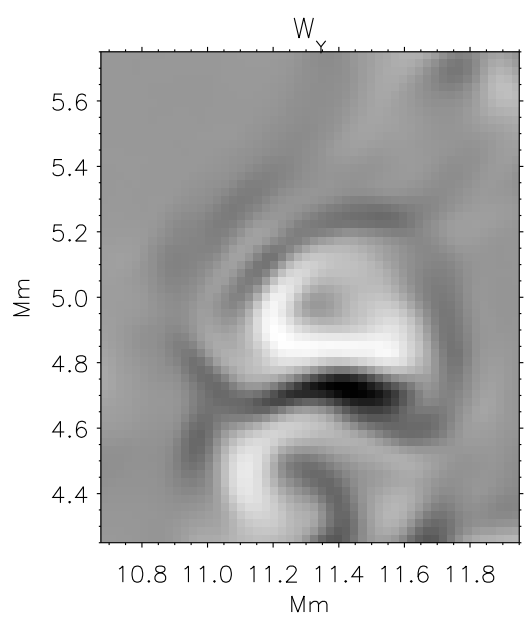

Fig. 5. Simulated G-band images of an MBP (top panels), vorticity at the visible solar surface (middle panels), and the vorticity in the upper photosphere (bottom panels).

vorticity generated due to the magnetic field is a factor of 4 larger than that generated by baroclinic motions in convecting photospheric non-magnetic plasma. By the appropriate decomposition of the MHD vorticity equation we defined two vorticity equation terms, which are connected to the magnetic field: the first resembles the baroclinic term in hydrodynamics, while the second contains the magnetic tension and does not have a direct hydrodynamic equivalent. We have demonstrated that it is only the latter term which is mostly responsible for the generation of vorticity in the upper photosphere. Conversely, the importance of the baroclinic hydrodynamic term increases with increasing geometrical depth. We also note the appearance of oscillations in the magnetic tension term in the upper photosphere. These may be signatures of the $3 \mathrm{mHz}$ lower-photospheric oscillations which leak to the upper layers through the intergranular magnetic field concentrations.

Using radiative diagnostics with the $\mathrm{G}$ band, we confirmed that MBPs are subject to rotary motions in the intergranular lanes and are magnetically connected to the vortices in the upper photosphere.

The large volume occupied by the intergranular vortices suggests their significance for the energy balance of the solar 
atmosphere. Further analysis is needed on the connection of the photospheric vortices with the chromospheric swirls, with the upper chromosphere and corona regions, and on torsional wave excitation in these regions of the solar atmosphere.

Follow-up investigations will focus on the details of the physical mechanism, which leads to the creation of negative and positive vorticity signs in a magnetic vortex. Extending the simulation box to include the chromosphere with a full non-LTE treatment of the radiative diagnostics (Carlsson et al. 2010), will also be the subject of a future investigation.

Acknowledgements. This work has been supported by the UK Science and Technology Facilities Council (STFC). F.P.K. is grateful to AWE Aldermaston for the award of a William Penney Fellowship.

\section{References}

Berdyugina, S. V., Solanki, S. K., \& Frutiger, C. 2003, A\&A, 412, 513

Bonet, J. A., Márquez, I., Sánchez Almeida, J., Cabello, I., \& Domingo, V. 2008, ApJ, 687, L131

Carlsson, M., Stein, R. F., Nordlund, Å., \& Scharmer, G. B. 2004, ApJ, 610, L137

Carlsson, M., Hansteen, V. H., \& Gudiksen, B. V. 2010, Mem. Soc. Astron. Ital., 81,582

Cheung, M. C. M., Schüssler, M., Tarbell, T. D., \& Title, A. M. 2008, ApJ, 687, 1373
Clyne, J., Mininni, P., Norton, A., \& Rast, M. 2007, New J. Phys., 9, 301 Danilovic, S., Schüssler, M., \& Solanki, S. K. 2010, A\&A, 513, A1

Emonet, T., \& Moreno-Insertis, F. 1998, ApJ, 492, 804

Emonet, T., Moreno-Insertis, F., \& Rast, M. P. 2001, ApJ, 549, 1212

Fedun, V., Erdélyi, R., \& Shelyag, S. 2009, Sol. Phys., 258, 219

Frutiger, C. 2000, Ph.D. Thesis No. 13896, ETH Zürich

Gruszecki, M., Nakariakov, V. M., van Doorsselaere, T., \& Arber, T. D. 2010, Phys. Rev. Lett., 105, 055004

Jess, D. B., Mathioudakis, M., Erdélyi, R., et al. 2009, Science, 323, 1582

Kitiashvili, I. N., Kosovichev, A. G., Wray, A. A., \& Mansour, N. N. 2010, ApJ, 719,307

Moreno-Insertis, F., \& Emonet, T. 1996, ApJ, 472, L53

Parker, E. N. 1988, ApJ, 330, 474

Pietarila Graham, J., Danilovic, S., \& Schüssler, M. 2009, ApJ, 693, 1728

Rempel, M., Schüssler, M., Cameron, R. H., \& Knölker, M. 2009, Science, 325, 171

Schüssler, M., \& Rempel, M. 2005, A\&A, 441, 337

Schüssler, M., Shelyag, S., Berdyugina, S., Vögler, A., \& Solanki, S. K. 2003, ApJ, 597, L173

Shelyag, S., Schüssler, M., Solanki, S. K., Berdyugina, S. V., \& Vögler, A. 2004, A\&A, 427, 335

Shelyag, S., Schüssler, M., Solanki, S. K., \& Vögler, A. 2007, A\&A, 469, 731

Solanki, S. K. 1987, Ph.D. Thesis No. 8309, ETH Zürich

Stein, R. F., \& Nordlund, A. 1998, ApJ, 499, 914

Vögler, A., \& Schüssler, M. 2007, A\&A, 465, L43

Vögler, A., Shelyag, S., Schüssler, M., et al. 2005, A\&A, 429, 335

Yelles Chaouche, L., Cheung, M. C. M., Solanki, S. K., Schüssler, M., \& Lagg, A. 2009, A\&A, 507, L53

Wedemeyer-Böhm, S. 2010, Mem. Soc. Astron. Ital., 81, 693

Wedemeyer-Böhm, S., \& Rouppe van der Voort, L. 2009, A\&A, 507, L9 